\title{
Multi-colour Imaging of Ultra-compact Objects in the Fornax Cluster
}

\author{
A. M. Karick ${ }^{1}$, M. D. Gregg ${ }^{1}$, M. J. Drinkwater ${ }^{2}$, M. Hilker ${ }^{3}$, and P. Firth ${ }^{2}$ \\ 1 University of California, Davis, USA, akarick@igpp.ucllnl.org \\ 2 University of Queensland, Australia \\ 3 Sternwarte der Universitat Bonn, Germany
}

Ultra-compact dwarf galaxies (UCDs) are a new type of galaxy we have discovered in the central region of the Fornax and Virgo clusters. Unresolved in ground-based imaging, UCDs have spectra typical of old stellar systems. Ninety-two have been found in Fornax [1][3], making them the most numerous galaxy type in the cluster. Although they form a cluster wide population, an over-density surrounds the central cluster galaxy, NGC1399, fueling controversy over their nature and origin. Several formation scenarios have been proposed. UCDs may be the remnant nuclei of tidally stripped $\mathrm{dE}, \mathrm{N}$ galaxies [2] or they may be the bright tail of the globular cluster (GC) population associated with NGC1399 [3, "UCOs"]. Alternatively they may be the first spectroscopically confirmed intracluster globular clusters (IGCs) in Fornax, resulting from hierarchical star cluster formation [4] and merging in intracluster space. The 5 brightest Fornax UCDs have M/L ratios indicating at lest some dark matter, unlike typical GCs.

\section{Multicolour Imaging of the Fornax Cluster}

We obtained deep multicolour $\left(\mathrm{u}^{\prime} \mathrm{g}^{\prime} \mathrm{r}^{\prime} \mathrm{i}^{\prime} \mathrm{z}^{\prime}\right)$ imaging of the central region of the Fornax Cluster using the CTIO 4m Mosaic Telescope. Fig. 1 (LEFT) shows the radial distribution of the present population of UCDs, GCs and $\mathrm{dE}, \mathrm{Ns}$. Sixty-two UCDs were discovered in our $2 \mathrm{dF}$ spectroscopic surveys of Fornax. An additional 30 were discovered in our recent VLT survey, from which candidates were pre-selected using this multicolour data [5]. Fig. 1 (RIGHT) shows the $\mathrm{g}^{\prime}-\mathrm{i}^{\prime}$ colour-magnitude relation (CMR) for the UCDs, UCOs [3], NGC1399 GCs [6][7][8], and cluster dwarf galaxies [9]. All objects are spectroscopically confirmed cluster members.

Bright UCDs and dEs follow similar CMRs: both populations become redder with increasing luminosity. The slope for the CMR for bright UCDs and fainter UCD candidates is $-0.05 \mathrm{mag}$, similar to the slope of the CMR of candidate UCDs ( $-0.07 \mathrm{mag}$ ) in Abell 1689 [10]. This is qualitatively consistent with UCDs being the stripped nuclei of dE,Ns. UCDs have similar colours to NGC1399 GCs, however they exhibit a larger spread in colour at $18<\mathrm{i}^{\prime}<20$, possibly reflecting a mixed metallicity or age spread. These results will be discussed in more detail in a forthcoming paper. 
At faint magnitudes, GC and UCDs cannot be distinguished by colour alone. High resolution spectroscopy to measure their internal velocity dispersions and metallicities, is needed to distinguish between GCs and UCDs. If dark matter is required to explain their dynamics then these faint compact objects are real galaxies or UCDs.
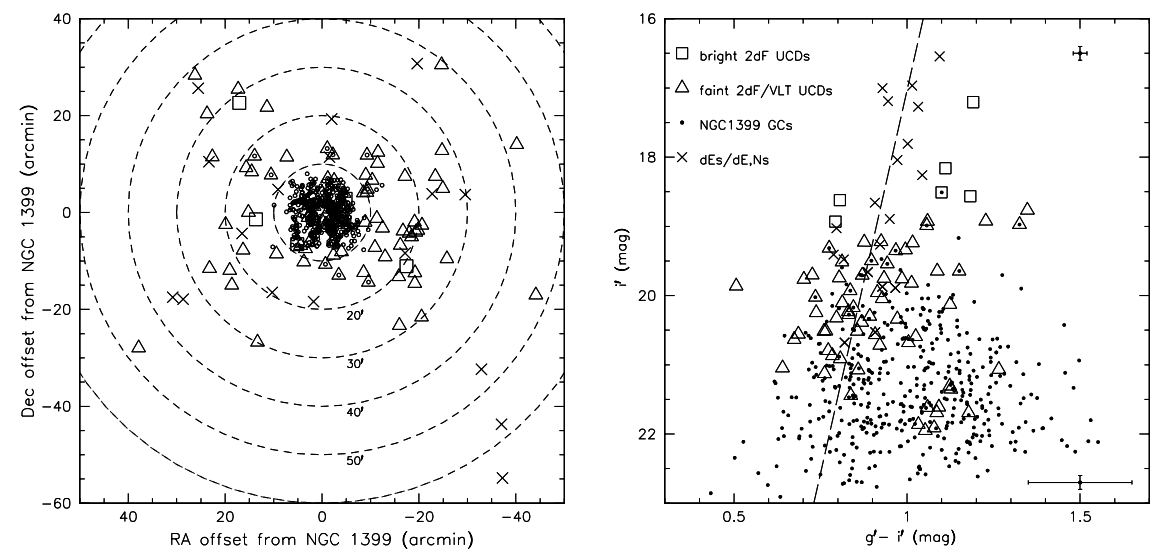

Fig. 1. LEFT: Distribution of compact objects surrounding NGC1399: bright 2dF UCDs (boxes), fainter 2dF/VLT UCDs (triangles), dE,Ns (crosses), NGC1399 GCs and UCOs (small circles). The NGC1399 GCs extend out to $10^{\prime}$ (survey limited) and overlap a small fraction $(7 \%)$ of the innermost UCDs. A significant number of the $2 \mathrm{dF} / \mathrm{VLT}$ UCDs (25\%) overlap the UCOs which extend out to $20^{\prime}$. RIGHT: $\mathrm{g}^{\prime}-\mathrm{i}^{\prime}$ CMR for compact objects in Fornax: 5 brightest 2dF UCDs (boxes), fainter 2dF/VLT UCDs (triangles), cluster dEs and dE,Ns (crosses) and NGC 1399 globular clusters (points). The dashed line shows the fit to the dwarf galaxy CMR. Typical error bars for $\mathrm{i}^{\prime} \sim 16.5 \mathrm{mag}$ and $\mathrm{i}^{\prime} \sim 22.5$ mag objects are also shown.

This project was supported by grants from the ARC and ANSTO Access to Large Research Facilities scheme. This material is based on part upon work supported by NSF grant no. 0407445 and carried out at IGPP under the auspices of the US DOE by LLNL under contract no. W-7405-Eng-48.

\section{References}

1. M. J. Drinkwater et al.: PASA 21, 375 (2004)

2. K. Bekki, W.J. Couch, M.J. Drinkwater: ApJ 552, 105 (2001)

3. S. Mieske, M. Hilker, L. Infante: A\&A 418, 445 (2004)

4. M. Fellhauer, P. Kroupa: MNRAS 330, 642 (2002)

5. A. M. Karick: PhD Thesis, University of Melbourne, Melbourne (2005)

6. B. Dirsch, et al.: AJ 127, 2114 (2004)

7. M. Kissler-Patig: AJ 115, 105 (1998)

8. D. Minniti, M. Kissler-Patig, P. Goudfrooij, G. Meylan: AJ 115, 121 (1998)

9. H.C. Ferguson: AJ 98, 367 (1989)

10. S. Mieske, L. Infante: AJ 128, 1529 (2004) 